SPARK CHAMBER NEUTRON AND PRCTON SPECTROMETER:

\title{
FIRST REPORT ON PERFORMANCES*
}

\author{
K. Mamont-Ciesia ${ }^{\dagger}$ and A. Rindi \\ Lawrence Berkeley Laboratory \\ University of Califormia \\ Berkeley, California 94720
}

December 17, 1974

\section{ABSTRACT}

An instrument has been built to measure neutron and proton spectra in the energy range between about 30 and $300 \mathrm{MeV}$ to be used in fields as $10 \mathrm{w}$ as about 0.1 particle $\mathrm{cm}^{-2} \mathrm{~s}^{-1}$. The software and harchare of the unit have been described elsewhere. $1, i$

We report on the checks and improvenents of some of the unfolding couputer prograns, on the measurements performed in a proton bean and in a neutron bean at the 184-Inch Synchrocyclotron, and on some measurements of stray proton spectra behind the shielding of the accelerator. We discuss finally the improvements that are needed for a full use of the potentialities of the instrument.
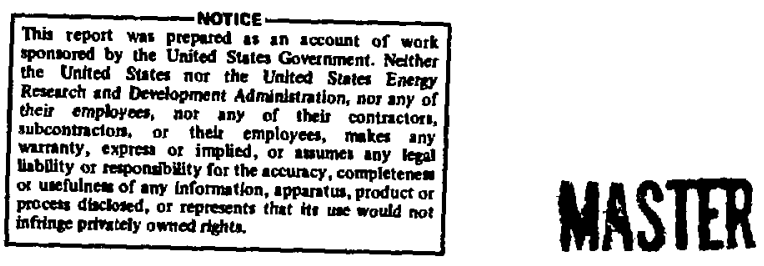

Work performed under the auspices of the U.S. Energy Research and Development Administration.

†Present address: Central Laboratory for Radiological Protection, Warsew, Poland. 
1. THE PROGRAM FOR UNFOLDING TIL PROTON SPECTRLM

The sensitive part of the detector is made of a sandwich of 12 multiwire spark chambers (50×50 $\mathrm{cm}^{2}$ dimensions), 15 plastic scintillators and 13 converter-absorbers of polyethylene $2.54 \mathrm{~cm}$ or $0.635 \mathrm{~cm}$ thick (see Fig. 1).

In the proton measuring mode, one event is a coincidence among the first three scintillators plus an anticoincidence with the last one, while in the neutron measuring mode it is a coincidence between at least two internal scintillators plus an anticoincidence with the first and last scintillators. Such an event, i.e., a proton entering the detector in the first case or a proton produced inside the detector by an incoming neutron in the second case, triggers the high voltage to the spark chambers. The coordinates of the sparks in the spark chimbers are recorded on a magnetic tape via a magnetostrictive readout system.

The computer program PTAPE reads from the tape the coordinates of the sparks for every event, calculates the length and the direction of the proton track, and, calculates the energy of the proton for a given thickness of absorber. It then plots a graph and punches cards of the number of protons versus energy and a table of angular direction versus energy.

In the neutron measuring mode from single scattered protons, the proton spectrum on punched cards, together with a matrix kernel and correction data calculated through a Monte Carlo program (DEER), provide the input for the program (LOUHI) which unfolds the neutron spectrum.

For the checking the precision of the program PTAPE we have modified its input such as to accept punched cards, and we have fed it with preconstructed sample events on punched cards.

For the use of the instrument as a neutron spectrometer from double scattering events, a modification of PTAPE has been written (called ASINO) which sorts from the magnetic tape only those events where the incoming neutron undergoes two consecutive proton scatterings inside the detector. It calculetes the scattering coordinates, track direction and energy of the two protons, and, from these data, calculates and plots the neutron spectrm and angular distribution. 


\section{MEASURDOUNS IN A PROTON BEAM}

A beam of $300 \mathrm{MbV}$ protons from the 184 -Inch Synchrocyclotron was extracted, collimated, and directed toward the front face of the spectrometer. It was used for timing the triggering scintillators. Then a block of: $5.3 \mathrm{~cm}$ of copper was introduced in the beam. This reduces the encrgy of the protons to about $163 \mathrm{MeV}$. 1000 odd events were recorded under these conditions. Then the beam attenuator was replaced with one of $4.3 \mathrm{~cm}$ thickness, which reduced the energy of the protons to about $193 \mathrm{MeV}$ and another 1000 events were recorded. The results of these measurenents as provided by MTAPE are shown in Fig. 2.

The width of the proton energy intervals for plotting the spectrum can be set to any value in the PTAPE program--it depends, of course, on the thickness of the absorbers and on the angular distribution of the protons. In Fig. 2 it is $8 \mathrm{MeV}$.

The figure shows the energy peaks at about 157 and $182 \mathrm{MeV}$. The small discrepancy with the calculated values can be because the exact energy of the prinary beam was not measured. If it was $295 \mathrm{MeV}$ instead of the assumed $300 \mathrm{MeV}$, the calculated and measured values coincide. (N.B. The calculation of the proton energy after crossing the copper attenuator was done using the formula

$$
E_{1}=\left[E_{2}^{1.64}-\frac{S}{7.27 \times 10^{-4}}\right]^{1 / 1.64}
$$

where $S$ is the copper thickness in $c m, E_{2}$ and $E_{1}$ are the proton energy in $\mathrm{MeV}$ before and after the attenuator respectively. This formula has been derived from an exponential range-energy relationship, i.e., of the type $R=K E^{a}$ ).

The results also show that the events were produced by proton whose tracks cross the spectrometer normally, i.e., that the spread of the beam at the attenuator was negligible.

Figure 2 confirms that the resolution that can be achieved in the spectraneter is dictated only by the thickness of the absorbers. 


\section{STRAY PROTON SPECTRA MEASURERENTS}

The spectrometer was positioned behind the shielding of the 184-Inch Synchrocyclotron, inside the accelerator building. The triggering logic was set as for triggering only on charged particles and spectra were taken for different experimental set ups at the accelerator. When we use this triggering logic there is still a probability of triggering on neutrons; this probability is the probability that tine neutron undergoes a $(n, p)$ reaction in the first scintillator. In Fig. 3 we plot this probability as a function of the $n$ energy for a plastic scintillator $0.32 \mathrm{~cm}$ thick. The discrininators of the triggering scintillators have only an upper threshold which was sot for $B$ rays from a $\mathrm{Ru}^{106}$ source. So we trigger on all the charged particles which have a $\mathrm{dE} / \mathrm{dx}$ higher than $-1.5 \mathrm{MeV} \mathrm{cm}^{2} \mathrm{~g}^{-1}$ in plastic scintillators. If a proton is scattered in the first scintillator, it must have an encrgy of at least $50 \mathrm{MeV}$ to be able to produce a pulse in the next two scintillators and generate a false triggering event. It can be produced only by neutrons of energy higher than $50 \mathrm{MeV}$. At this energy the probability of scattering a proton in the first scintillator is $\sim 2 \times 10^{-3}$. Then the number of spurious events produced by neutrons to protons is lower than about 500:1 :in such a field one can expect to have some $30 \xi$ of events produced by neutrons.

The triple coincidence rate during the measurements was about 10 events per second. This rate for the given dimensions of the scintillators $\left(50 \times 50 \mathrm{~cm}^{2}\right)$ is produced by a field of $4 \times 10^{-3}$ particles $\mathrm{cm}^{-2} \mathrm{~s}^{-1}$. A BF 3 moderated neutron counter, which is sensitive to neutrons of energy between about $100 \mathrm{keV}$ and $5 \mathrm{MeV}$, was set besicie the spectrometer and indicated a field of about $5 \mathrm{n} \mathrm{cm}^{-2} \mathrm{~s}^{-1}$. Assuming a neutron spectrum of the $1 / E$ type, one can infer that there were not more than about $0.5 \mathrm{n} \mathrm{cm}^{-2} \mathrm{~s}^{-1}$ of energy higher than 50 NeV. The ratio of neutrons to protons was, then, about 100:1. We can conclude that the number of spurious events in the spectra was very small.

Figure 4 shows a proton spectrum recorded when a 75" teV p beam, extracted from the 184-Inch Synchrocyclotron, was stopped in a cu target 10cated inside an experimental cave. The spectrometer was located at about $90^{\circ}$ fro the bean direction, some $20 \mathrm{~m}$ from the main target; the thickness of the concrete wall of the cave in the direction of the spectroneter was about $4 \mathrm{~m}$. The angular distribution of the detected protons indicates the target as the 
main source.

Figure 5 shows a spectrum taken from the same position. This time the extracted $750 \mathrm{MeV}$ p bean was mainly stopped in a concrete beam stopper at the end of the cave; the experimental targets were absorbing only a small fraction of the bean.

The slopes of the curves of Figs. 4 and 5 are about the same. The curves seem to fit quite well an equation of the type $N=K_{1} e^{-K_{2} E}$ where $N$ is the number of protons $E$ is their energy, and $K_{1}, K_{2}$ are constants.

Figure 6 shows a spectrum taken when the $670 \mathrm{MeV}$ proton bean was stopped in a target inside the accelerator. In this meusurement the secondary particles had to cross the iron of the magnet yoke and the external $3 \mathrm{~m}$ concrete shielding before reaching the spectrometer.

Several authors have performed Monte Carlo calculations of charged particle spectra produced by high energy proton beans and transmitted through different materials (see, for instance Refs. 3,4). In particular, J. Routti has kindly perfomed the calculation of the transport of the cascade in an Al block using the computer program TRANKA. 5

The shape of the spectra we have measured seems to fit the theoretical calculations. However, our experinental geometrical conditions were not precisely defined. We are planning to perform other measurements under more controlled experimental conditions, such that comparison's with calculated results can be unambiguously made. In addition, the absolute proton and neutron fluences have to be evaluated.

We are also planning to improve the spectroneter design. The rate of event recording is limited to about 1 event per second by the tape recorder; the gas and high voltage supplies for the spark chambers and the triggering scintillators make the apparatus rather heavy and cumbersome.

In view of its possible application for measuring spectra at different altitudes in airplanes, we are planning to replace the multiwire spark chambers with sealed multiwire proportional chambers which will elininate the necessity of triggering scintillators.

We want to thank Dr. Ralph H. Thomas for his continuous encouragement and his help in measuring the proton spectra at the 184-Inch Synchrocyclotron. 


\section{REFLREXCES}

1. A. Rindi, "A spectroneter for measuring charged particles and neutrons," Nuc1. Instr. and Meth. 116, 471 (1974).

2. C. B. Lim, "The developnent of a neutron spectrometer using llultiwire spark chambers for the measurement of the spectra of stray neutrons in the vicinity of high energy accelerator" (Ph.D. 'Mhes is, Unuversity of California, 1973), Lawrence Berkeley Laboratory Report, I,RL-1719.

3. J. Ranft, "Estimation of radiation problems around high energy accelerators using calculations of the hadronic cascade in matter," Particle Accelerators 3, 129 (1972).

4. R. G. Alsmiller, J. W. Wachter, and II. S. Mbran, "Ca1culation of the neutron and proton spectra from thick targets bombarded by $450 \mathrm{MeV}$ protons and comparison with experiment," Nucl. Science Engir. 36, 291 (1969).

5. J. Routti, Private communication, unpublished. 


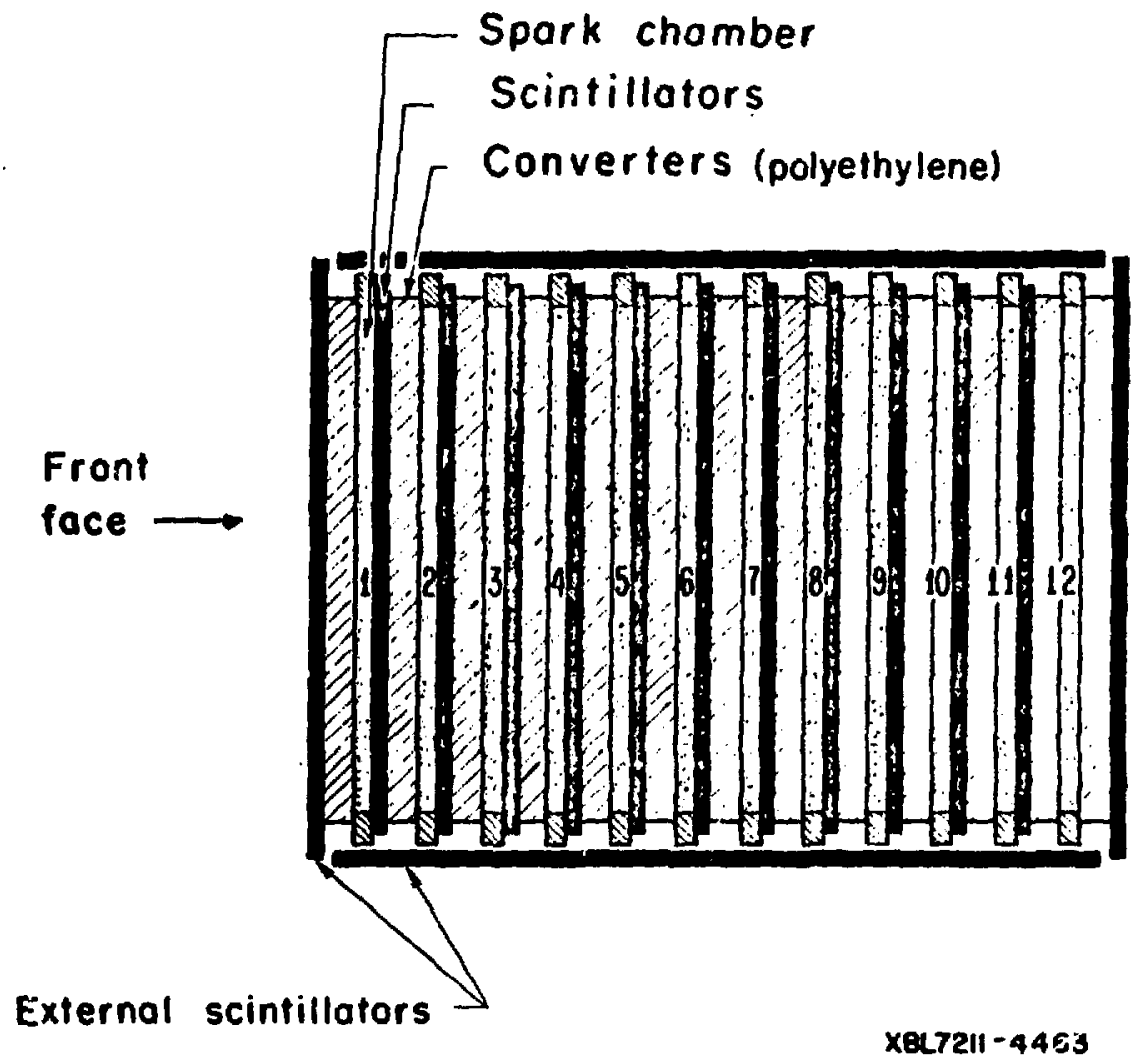

Figure 1. A schematic view of the sensitive part of the spectrometers. 


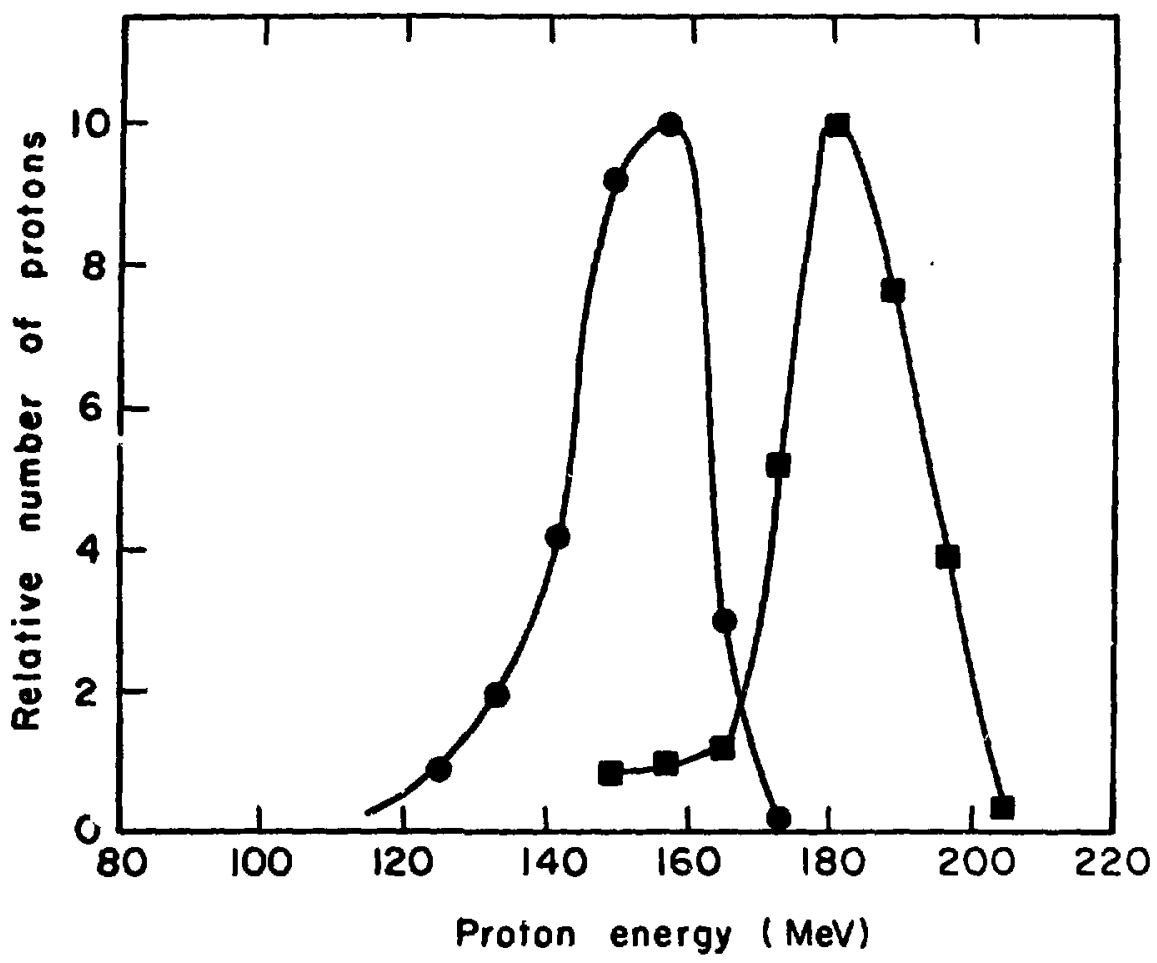

XEL751-2104

Figure 2. Spectrum of proton measured in the $300 \mathrm{MeV}$ proton beam of the 184-Inch Synchrocyclotron, attenuated in $5.3 \mathrm{~cm}$ (peak at left) and $4.3 \mathrm{~cm}$ of copper (peak at right). 


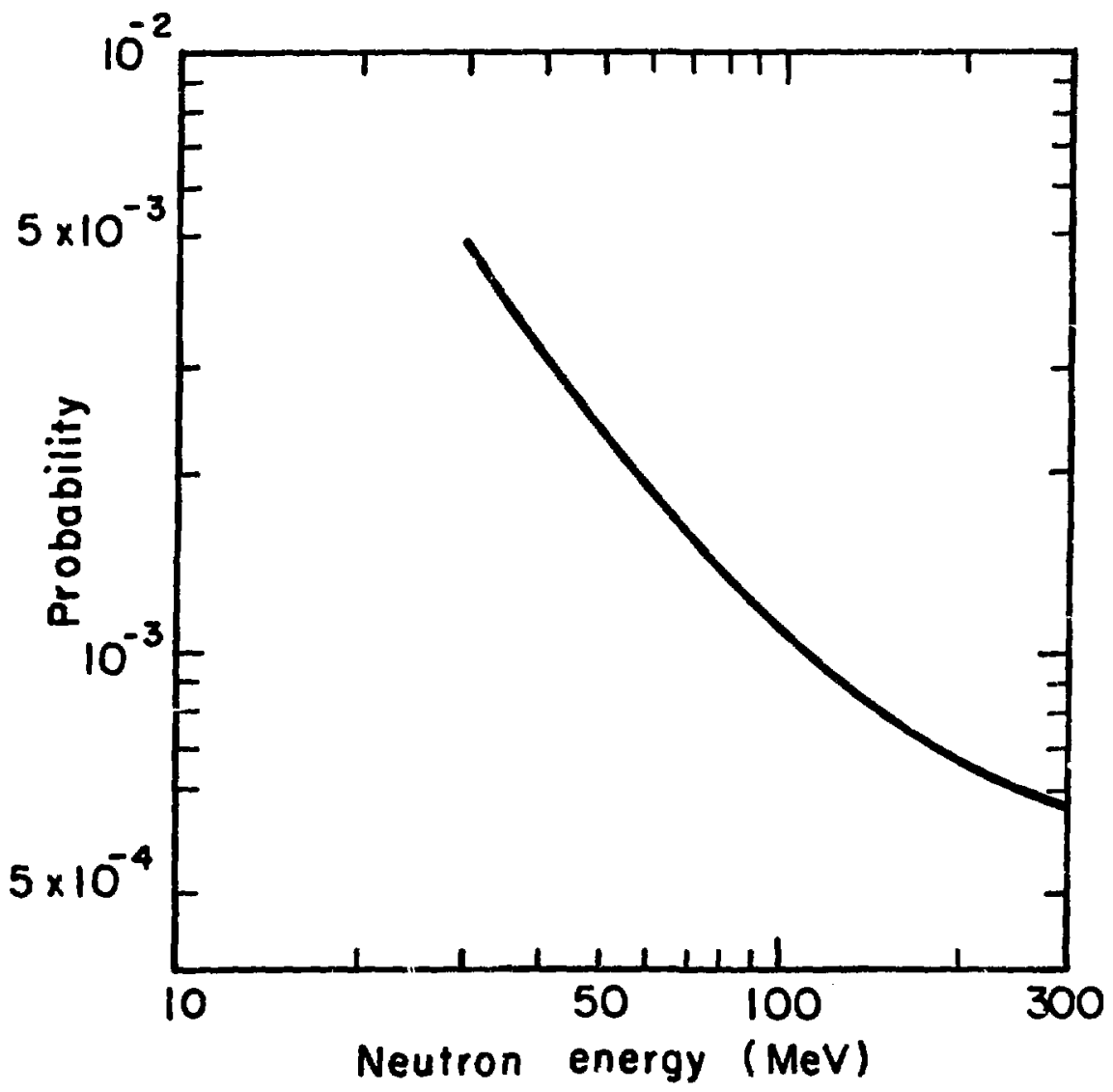

XBLT51- 2006

Figure 3. The probability that a neutron produces a recoil proton in the first triggering scintillator as a function of the neutron energy. 


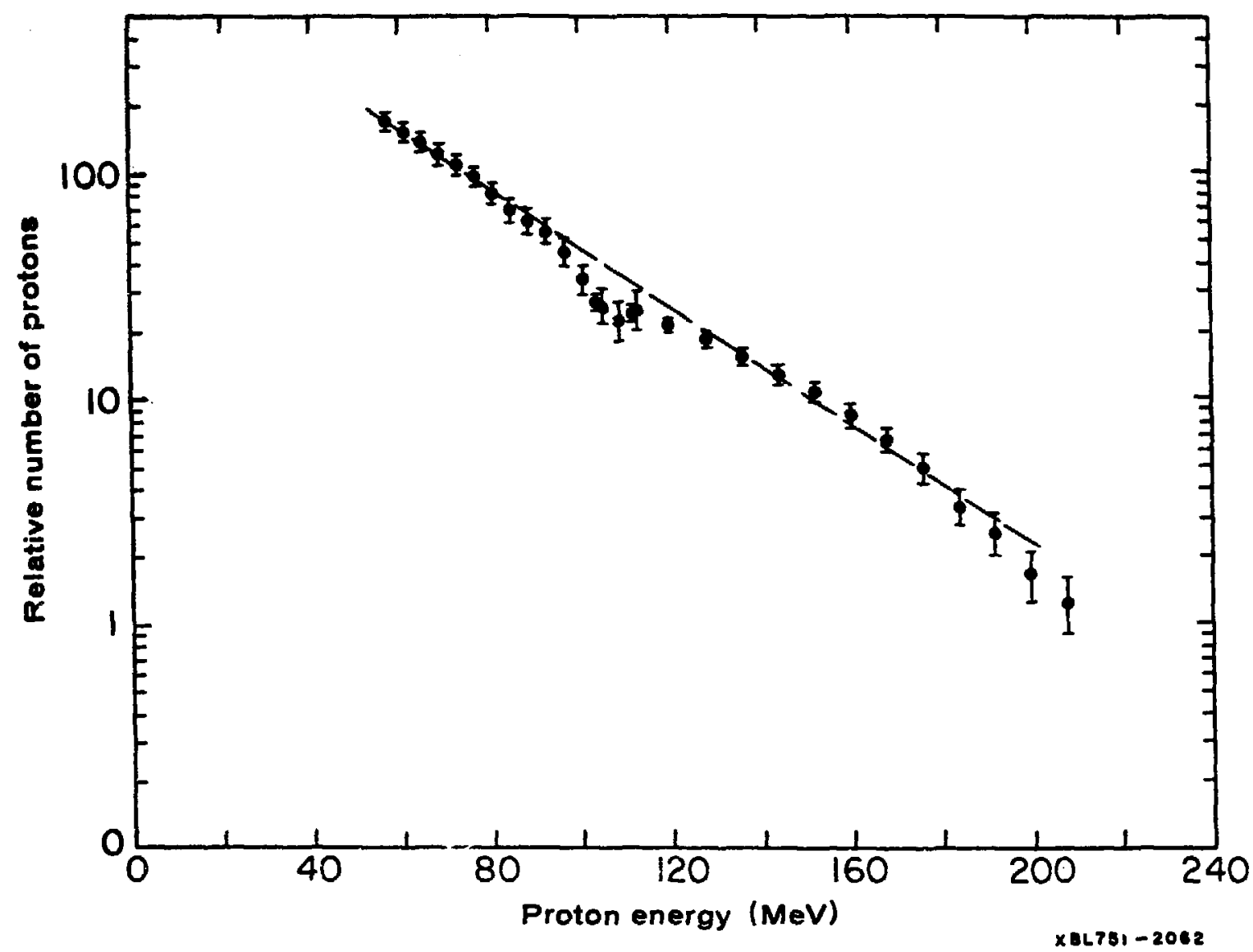

Figure 4. Proton spectrum measurec in the building of the 184-Inch Synchrocyclotron when a $750 \mathrm{MeV} p$ beam was stopped in a Cu target inside a shielded experimental cave (proton cave). 


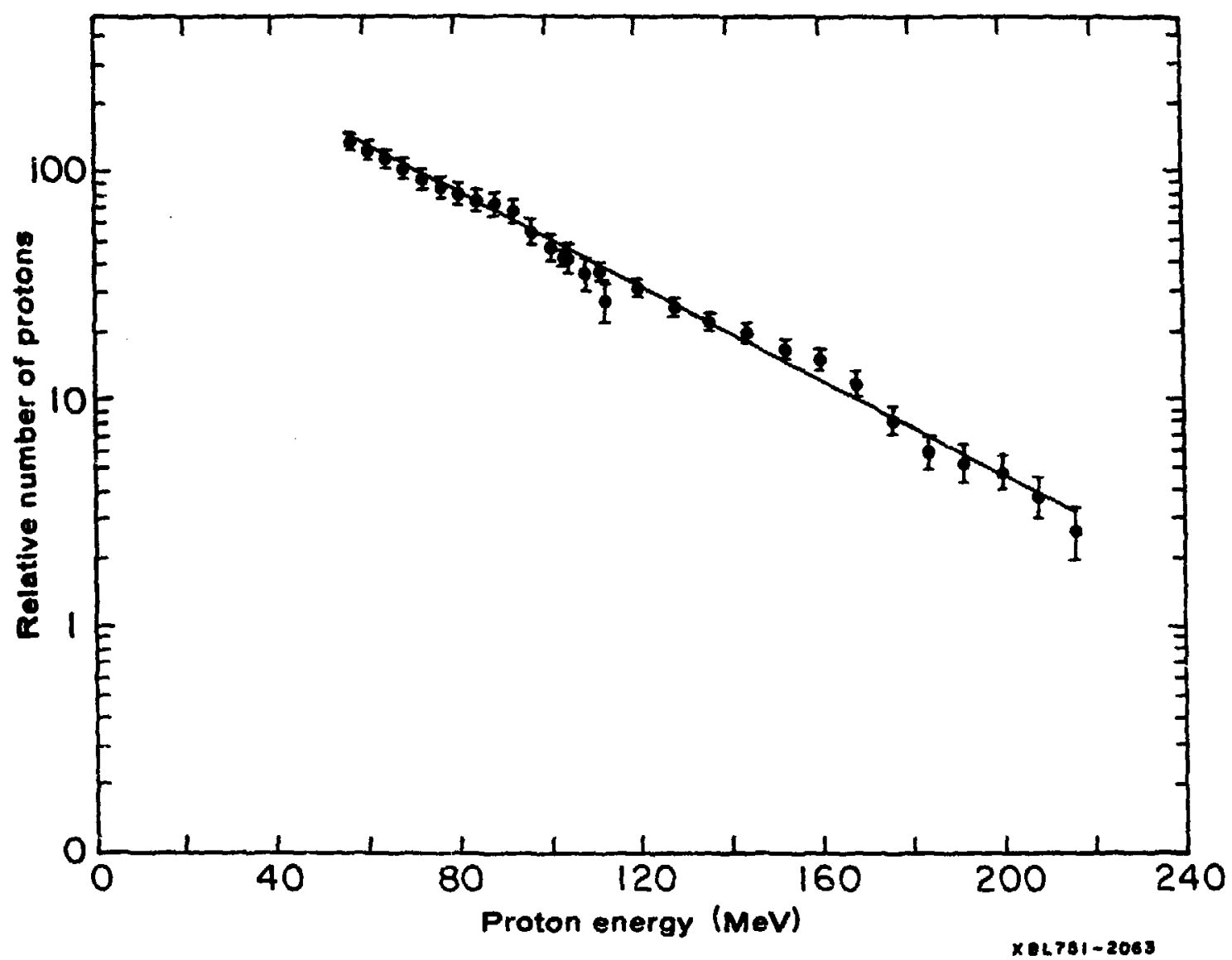

Figure 5. Proton spectrum measured in the building of the 184-Inch Synchrocyclotron when a $750 \mathrm{MeV}$ proton bean was stopped in a concrete beam stopper inside a shielded experinental cave (proton cave). 
Relative number of protons

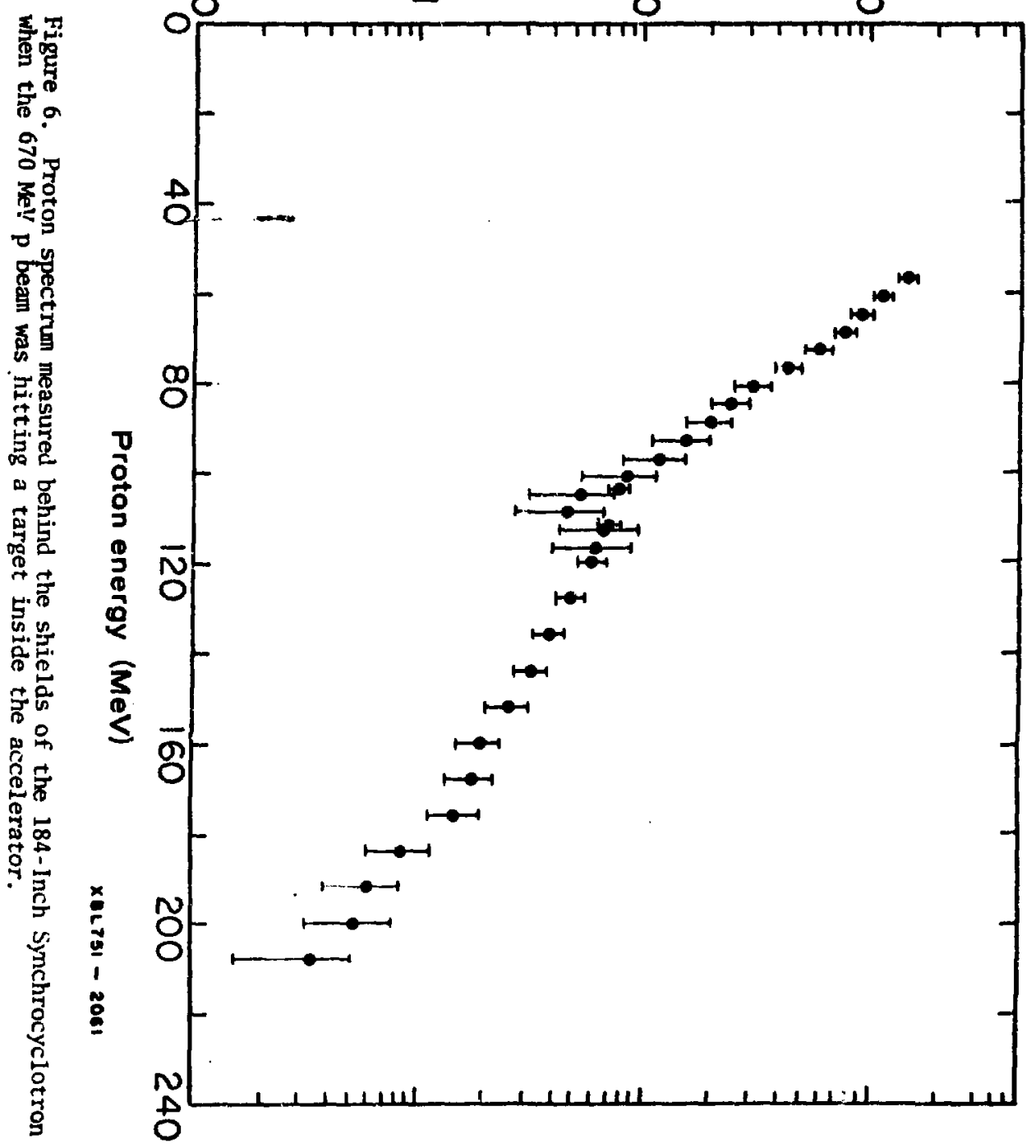

